\title{
The application status and prospect of ant colony algorithm
}

\author{
Guo Jian ${ }^{1,}$ a, Zhou Beiduo ${ }^{2, b}$ \\ ${ }^{1}$ Office of teaching affairs, Beijing Wuzi University, Beijing, 101149, \\ China \\ ${ }^{2}$ Graduate faculty, Beijing Wuzi University, Beijing, 101149, China \\ a email: guojian760118@163.com, ${ }^{b}$ email:2009duoduo.ok@163.com
}

\begin{abstract}
Ant colony algorithm is one of the representative methods of swarm intelligence. It is a kind of simulation optimization algorithm. Because it has many good qualities, many NP - hard problems have been solved successfully by using it. This article first expounds the basic theory of ant colony algorithm and makes the traveling salesman problem (TSP) an example to illustrate the basic model of ant colony algorithm. Then it summarized its application in static and dynamic combination optimization problem. Finally, the article makes the prospect of the direction of ant colony algorithm in research and application.
\end{abstract}

Keywords: Ant colony algorithm; application status; prospect

\section{Introduction}

Ant colony algorithm or ant algorithm comes from random search in bionics. It is a bionic optimization algorithm after the genetic algorithm, simulated annealing algorithm, taboo search algorithm, artificial neural network algorithm and so on. It is proposed by the Italian scholars Dorigo $\mathrm{M}$ and others who were inspired by the real ants' collective behavior. With its excellent properties, many combinatorial optimization problems have bean solved by using it. Since it is widely used nowadays, the paper made a summary of its application status and prospect.

\section{The basic principle and model of ant colony algorithm}

The studies of biologists have showed that although a single ant's memory and intelligence is very limited, groups of ants can realize the information communication and pass by the synergistic effect of pheromone, showing astonishing swarm intelligence behavior. In the following, the paper illustrated the basic principle of ant colony algorithm. The paper made how group ants found the shortest route between the nest and the food through synergistic effect an example.Then, the paper made the TSP problem as an example to illustrate the basic model of ant colony algorithm. 
2.1The basic principle of ant colony algorithm

We assumed that there are two paths from the ant nest to the food source, the length of them are 4 and 6 as shown in figure 1.The amount of pheromone on both paths are 0 in the beginning, regardless of the pheromone volatilization. Then we assumed that if the ants past once, the pheromone of the pass will increase 1 and the ants can only move a unit of length in one second.

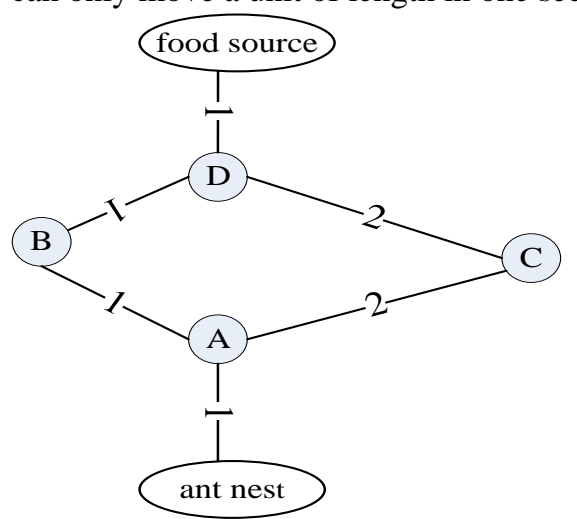

Figure.1 the diagrammatic presentation of ants from ant nest to food source

At the time of zero, 20 ants started moved to the food source, they choose the path A-B-D and path A-C-D inthe same probability, so there are 10 ants choose the path A - B - D and 10 ants path A - C - D.

At the time of four, the group first arrived would return, and at the same time the second group arrived at the midpoint of CD.

At the time of five, the two groups met at point D. At this moment, the amount of pheromone on the BD and CD are the same. So there are 5 arrived ants chosen $\mathrm{BD}$ and the other five chosen $\mathrm{CD}$. The second group continued moving to the direction of the food.

At the time of eight, five ants would reach the nest. At this time, there were five ants respectively at the midpoint of the AC, $\mathrm{CD}$ and point $\mathrm{B}$.

At the time of nine, there are five ants return to $A$ and again in the face of choice path A - B - D or A - C - D.

At this time, the amount of pheromone on path AB was 20 while AC was 15. Therefore, there would be more ants choose the path of $A-B-D$, thus the pheromone on this path was increased. With the process continued, the pheromone difference on the two paths becoming bigger and bigger. In the end, the majority of ants have chosen path A - B - D. This was the principle by which groups of ants found the shortest path from the nest to the food source.

2.2The basic model of ant colony algorithm

Since the traveling salesman problem was a classic combinatorial optimization problem, and the ant colony algorithm was first used in traveling salesman problem (TSP). So the paper made the TSP problem as an example to illustrate the basic model of ant colony algorithm. Below was the simple description of TSP. Among the several given cities, we made the distance between city $\mathrm{i}$ and $\mathrm{j}$ as $\mathrm{d}_{\mathrm{ij}}$. Then we chose one as the starting point of a traveling 
salesman. Each city can only be passed once. After traveled all the cities, he returned to the starting point. We need to find the shortest path.

Supposed that the numbers of ants were $m$ and every ant choose the next city according to the distance between the two cities and the amount of the pheromone .The probability that shown ant k moved from city i to city j was $P_{i j}^{k}$. Its formula was as follows:

(1)

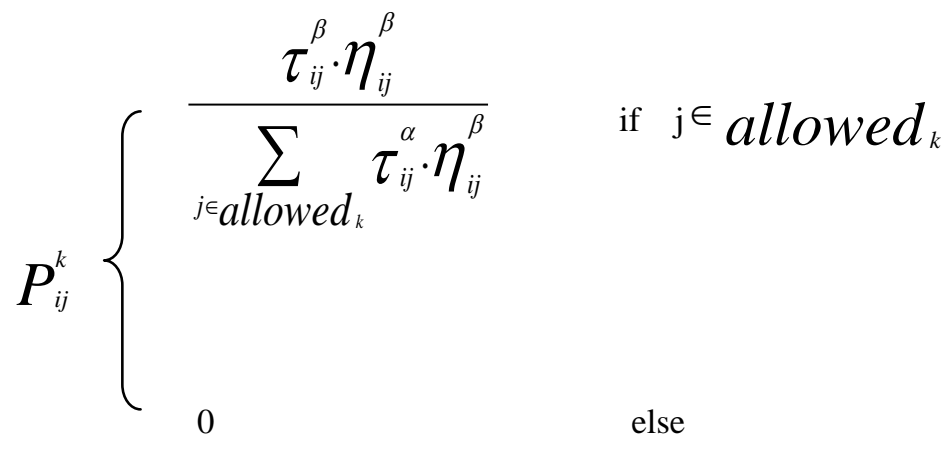

In the formula, $\tau_{i j}(t)$ means that the density of pheromone along the path from the city i to city $\mathrm{j} ; \boldsymbol{\eta}_{i j}$ means the heuristic information along the path from the city $\mathrm{i}$ to city $\mathrm{j}$ and $\eta_{i j}$ equal the reciprocal of $\mathrm{d}_{\mathrm{ij}} ; \alpha$ means the heuristic information factor; $\beta$ means the expect heuristic factor;

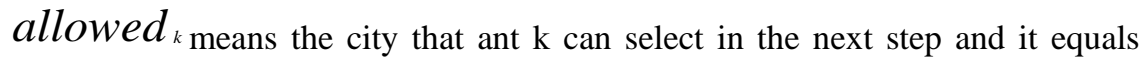
$\{0,1,2 、$, $n-1\}$ - tabuk; tabuk means the taboo table of ant $\mathrm{k}$ in which record the cities that ant $\mathrm{k}$ have pass.

Since pheromones are volatile, the pheromone on the path should be updated after an ant in a loop. The update formulas are as follows:

$$
\left\{\begin{array}{l}
\tau_{i j}(t+1)=(1-\rho) \tau_{i j}(t)+\hat{t}_{i j} \\
\Delta \tau_{i j}=\sum_{k=1}^{m} \tau_{i j}^{k}
\end{array}\right.
$$

$\boldsymbol{\rho}$ is Often referred to pheromone volatilization factor, $\rho \in[0,1]$; $\Delta \tau_{i j}$ is the increment of the pheromone, $\tau_{i j}^{k}$ means the information on path $\mathrm{ij}$ that the ant $\mathrm{k}$ leaved in one cycle, its formulas are as follows: 


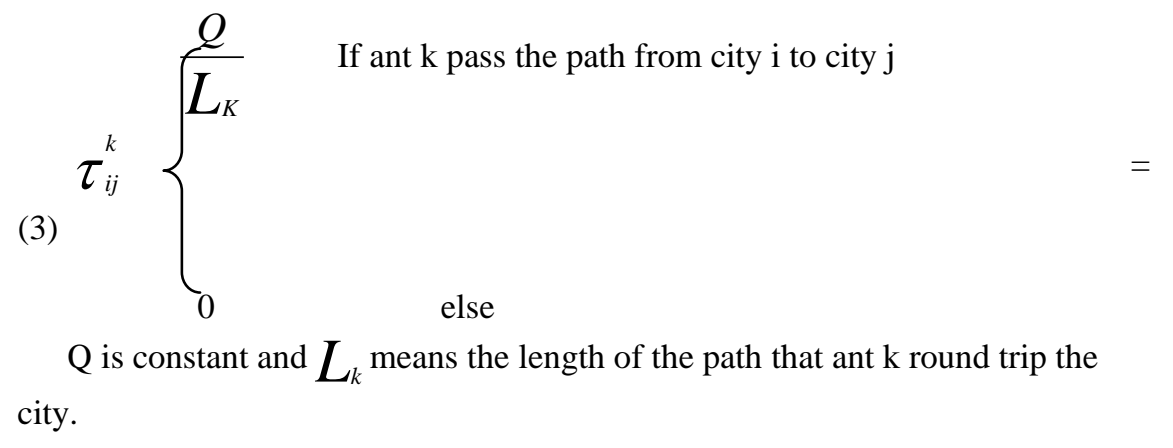

\section{The present application status of ant colony algorithm}

At present, the applied research of ant colony algorithm is mainly concentrated in two aspects, the static combinatorial optimization problem and dynamic combinatorial optimization problem. Dynamic combinatorial optimization problem is that the problems are changing in the performance period and optimization algorithm must be adapted to the changing environment. Such as the typical network routing problem and so on.

3.1 The application of Ant colony algorithm in static combinatorial optimization problem

Static combinatorial optimization problem is that the characteristics of the problem were given in one-time and they won't change in the process of solving the problem. Such as the classical traveling salesman problem (TSP), the quadratic assignment problem (QAP), the Job-shop scheduling problem (JSP), vehicle routing problem (VRP) and so on.

3.1.1 The application of Ant Colony Algorithm in traveling salesman problem

As the basic ant colony algorithm have local optimum and slow convergence speed problems in solving TSP. Recent years, many scholars make improvement for it.

In our country, Pei-dong Wang (2013) [1] and other scholars have avoided the algorithm to a standstill state by combining local pheromone updating and global dynamic updating method to update pheromone in the current optimal path. To accelerate the algorithm convergence speed, they only used 2 - opt method to the travel businessmen who walked better path. The simulation experimental results showed that the algorithm have a good performance both in the solution quality and convergence rate. Hua-feng Wu (2013) [2] and other scholars proposed a modified ant colony algorithm based on natural selection strategy. According to the experimental results, the algorithm in the optimal solution accuracy and convergence speed was both improved. Foreign scholars Yang Jinhui (2008) [3] and others proposed an ant colony algorithm with variation process and local search. TSP problems could be solved well by using this improved ant colony algorithm.

3.1.2 The application of Ant colony algorithm in quadratic assignment problem

Quadratic assignment problem (QAP) is the second successful application of 
ant colony algorithm after the TSP problem. Quadratic assignment problem means that choosing several positions, and then assigns several factories to be built in each one accordingly. We should find how to allocate to make the cost the least. In the question, the cost is the function that the factory assigned to the position.

As the basic ant colony algorithm has the characteristic of distributed parallel search, it can solve small secondary distribution problems easily. With the quadratic assignment problem expanding, it is difficult to find the optimal solution in limited times by using basic ant algorithm. Therefore, it was improved by many scholars.

Jing-wei Zhu (2011) [4] and other scholars, proposed a simulated annealing ant colony algorithm by introducing simulated annealing mechanism into the ant colony algorithm. And the experiment showed that the algorithm has high stability and convergence speed. Dong-feng Yuan (2013) [5] and other scholars, introduced the local optimization algorithm into the basic ant colony algorithm and using vertex relocation of local optimization algorithm to improve each optimum generation in order to speed up the convergence speed of the algorithm. Compared with the basic ant colony algorithm, the algorithm has better performance.

3.1.3 The application of ant colony algorithm in shop scheduling problem

Job-shop scheduling problem (JSP) means that using several machines to process some work pieces and each piece has $\mathrm{k}$ processing procedures, each procedure should be processed in a certain order on different machines, and each machine can only process one piece in a time. The problem we need to handle is that how to allocate the order in each machine to make the goals best.

Yanhong Wang (2013) [6] and other scholars proposed a dynamic balance adaptive ant colony algorithm in order to deal with the problems of falling into local optimum and long search time which emerge when solving work shop scheduling problems. Results showed that the improved algorithm work better not only in convergence speed, but also in solution quality and solution stability.

Foreign scholars, Betul Yagmahan (2009) [7]and other scholars proposed a multi-objective ant colony algorithm by combining the basic ant colony algorithm with local search algorithm .The experimental results showed that the algorithm is better than other algorithms in solving job-shop scheduling problem whose goals are minimizing the makespan and the total completion time .

3.1.4 The application of ant colony algorithm in vehicle routing problem

Vehicle routing problem (VRP) means that several vehicles delivering goods from the distribution center to customers according to their demanding amount. The goal is how to achieve the certain purpose in the constraint conditions and meet the needs of customers. The purpose include the shortest distance, the least costs, the minimum time etc.

Vehicle routing problem is an important part in the optimization processes of distribution center. It has attracted scholars to study for a long time. Because the basic ant colony algorithm have problems of slow convergence speed and long operation time and it is easy to fall into local optimum in solving Vehicle routing problems, many scholars have made improvement in it. 
Domestic scholars, Yingxin Chen(2012)[8],improved the pheromone updating strategy and the heuristic factor in basic ant colony algorithm. He proved the feasibility and effectiveness of the improved algorithm through experiment, aiming at improving the defects of ant colony algorithm. Foreign scholars, Zhang Xiao (2012) [9] and others, proposed a hybrid ant colony algorithm in order to improve the quality and performance of ant colony algorithm in solving the vehicle routing problems. Result showed that it was effective in solving vehicle routing problems.

3.2The application of ant colony algorithm in dynamic combinatorial optimization problem

The feature of Dynamic combinatorial optimization problem is that the solution of the question is changing with the time. The typical example is network route which can be divided into directed connection and connectionless system. The directed connection route means that packets of the same speech path transferred along a common path which is chosen by the state of the preliminary set state. Instead, the connectionless network route means that the network data packets from the same speech path can be transmitted along different paths. How to transmit from the source node to the end is decided by the local routing components [10]

Recent years, the application of multimedia emerge in endlessly with the rapidly development of modern networks. As the traditional transport services cannot completely meet the demand of multimedia business data transmission, the quality of service (QoS) routing becomes a growing trend.

As to the question of the low rate of access and system switch, domestic scholars, Xiaoxue Guo (2010) [11] and others proposed a dynamic hierarchical bandwidth allocation, QoS link access control algorithm. The experimental results showed that the algorithm is successful in access and total system bandwidth utilization and system performance. It is better than traditional link access control algorithm, and it is suitable for the large-scale application of broadband access link control.

\section{Prospects of the research and application of ant colony algorithm}

Although there are many scholars studying ant colony algorithm, it is still not mature compared with other intelligent algorithm, further research is needed. Its research and application prospects are as follows.

4.1 The research prospect of ant colony algorithm

First, the ideal ant colony algorithm which assumed that the ants had not met enemies in the process of optimization is opposite of the reality. We can add enemies' disturb direction to improve the ant colony algorithm.

Second, the theory basis of ant colony algorithm is not strong at present, so we should strengthen the theory study of ant colony algorithm.

Third, there is only the study of one intelligent algorithm combination with ant colony algorithm now. In the future, we should combine more intelligent algorithms with ant colony algorithms to make full use of the advantages of 
them.

Fourth, the initialization of parameters in ant colony algorithm is mostly based on experience and it is not standardized, so in the future, we should make studies of the setting of these parameters' initialization.

Fifth, the model of ant colony algorithm goes not universally, therefore, we should increase the general study of ant colony algorithm.

4.2 The application prospect of ant colony algorithm

Since the ant colony algorithm has the advantages of simple realization forms, distributed positive feedback, so it has been widely used. Ant colony algorithm can solve problems in many fields. Its prospects are as follows:

On one hand, there is no scholar applies the ant colony algorithm to financial engineering currently, so we can consider applying the ant colony algorithm into the development of financial system of financial engineering.

On the other hand, with the rapid development of e-commerce, the distribution center plays a more and more important role; the ant colony algorithm can be served to the distribution center in the future.

\section{Concludes}

This paper introduces the basic principle of ant colony algorithm. Then it summarized the application of ant colony algorithm in static combinatorial optimization problems and dynamic combinatorial optimization problems. Finally, the paper makes an outlook of ant colony algorithm in the direction of the research and the application. First, we can add enemies to improve the ant colony algorithm. Second, the research on the theory of ant colony algorithm should be increased. Third, ant colony algorithm can be combined with a variety of intelligent algorithm. Fourth, study how to initialize the setting parameters of ant colony algorithm. Fifth, apply the ant colony algorithm to the financial engineering field. At last, apply the ant colony algorithm to the distribution center service. Finally, the paper summarized the application status and prospect of ant colony algorithm as well as the way for the further studies of ant colony algorithm.

\section{Acknowledgement}

In this paper, the research was sponsored by Funding Project for Science and Technology Program of Beijing Municipal Commission of Education

( KM201310037001 ) ,Beijing Youth Talent Development Plan

( CIT\&TCD201504052 ) ,Beijing Wuzi University Youth Yunhe

Scholar,Beijing Wuzi University Major Program. 


\section{References}

[1]Wang Peidong,Tang Gongyou,Yang Xixin, Li Yang. An improved ant colony algorithm to solve traveling salesman problem. Journal of ocean university of China ,2013,43 (1) : 93-97(in Chinese)

[2]Wu Huafeng,Chen Xinqiang,Mao Qifeng,Zhang Qiannan,Zhang Shouchun. Ant colony algorithm that based on natural selection strategy to solve TSP problem. Journal of communication,2013,34 (4):165-170(in Chinese)

[3]Yang J H,Shi XH,Marchese M,et al.An Ant Colony Optimization Method for Generalized TSP Problem[J].Progress in Natural Science,18(2008):1417-1422.

[4]Zhu Jingwei,Bing Ting, Jiang Xinsheng,Zhang Jinlin. Simulated annealing ant colony algorithm for solving quadratic assignment problem,2011,47 (14) :34-36(in Chinese)

[5]Yuan Dongfeng, Lv Congying. The application in quadratic assignment problem of improving ant colony algorithm. Computer and modern,2013,03 (03):9-11(in Chinese)

[6]Wang Yanhong,Wang Wenxia,Yu Hongxia,Chen Li. Dynamic balance adaptive colony algorithm solving Job-Shop scheduling,2013,10 (19): 2521-2527(in Chinese)

[7]Betul Yagmahan,Mehmet mutlu Yenisey.A multi-objective ant colony system algorithm for flow shop scheduling problem[J].Expert Systems with Applications,37(2009):1361-1368.

[8]Chen Yingxin. The research of optimizating Vehicle routing problem based on improved ant colony algorithm. Computer application research,2012,29

(6) :2031-2034(in Chinese)

[9]Zhang Xiao,Wang Jing-Qing. Hybrid Ant Algorithm and Applications for Vehicle Routing.Problem[J]. Physics Procedia 25 ( 2012 ) :1892 - 1899

[10]Deng Yufen,Xiang Fenghong. The application of ant colony algorithm in combinatorial optimization problem. Electronic measurement techniques,2007,30 (1):32-35(in Chinese)

[11]Guo Xiaoxue,Qin Yong,Ye Jianfeng. Dynamic Bandwidth Allocation and Multi-level QoS. Computer engineering and application,2010,46(15):8l 84(in Chinese) 